\title{
Macrocell Protection Interference Alignment in Two-Tier Downlink Heterogeneous Networks
}

\author{
Jongpil Seo, Hyeonsu Kim, Jongmin Ahn, and Jaehak Chung \\ Department of Electronic Engineering, Inha University, Incheon, Republic of Korea \\ Correspondence should be addressed to Jaehak Chung; jchung@inha.ac.kr
}

Received 1 August 2016; Revised 4 November 2016; Accepted 1 December 2016; Published 3 January 2017

Academic Editor: Mariusz Głąbowski

Copyright (C) 2017 Jongpil Seo et al. This is an open access article distributed under the Creative Commons Attribution License, which permits unrestricted use, distribution, and reproduction in any medium, provided the original work is properly cited.

\begin{abstract}
Conventional interference alignment (IA) has been developed to mitigate interference problems for the coexistence of picocells and macrocells. This paper proposes a macrocell protection interference alignment (MCP-IA) in two-tier MIMO downlink heterogeneous networks. The proposed method aligns the interference of the macro user equipment (UE) and mitigates the interference of the pico-UEs with a minimum mean squared error interference rejection combining (MMSE-IRC) receiver. Compared to the conventional IA, the proposed MCP-IA provides an additional array gain obtained by the precoder design of the macro BS and a diversity gain achieved by signal space selections. The degrees of freedom (DoF) of the proposed MCP-IA are equal to or greater than that of the conventional IA and are derived theoretically. Link level simulations show the link capacity and the DoF of the macro UE, and also exhibit the proposed MCP-IA attaining additional array gain and diversity gain. The system level simulation illustrates that the proposed method prevents the interference of the macro UE completely and preserves the throughput of the pico-UE irrespective of the number of picocells. For $4 \times 2$ antenna configuration, the system level simulation demonstrates that the proposed MCP-IA throughput of the macro UE is not affected by the number of picocells and that the proposed MCP-IA throughput of the picocells approaches that of single-user MIMO (SU-MIMO) with a 3\% loss.
\end{abstract}

\section{Introduction}

Heterogeneous networks have been researched for improving the system capacity [1-3]. Pico-, femto-, or relay base stations (BSs) are adopted to cover the shadowing regions and increase the system capacity with a lower power compared to that of the traditional macro BSs. In heterogeneous networks, all the networks utilize the same carrier frequency to increase the spectral efficiency and to avoid bandwidth segmentation. Severe intercell interference at the boundaries of the small cells, however, reduces the system capacity [3].

To overcome intercell interference, enhanced intercell interference coordination (eICIC) techniques were developed $[4,5]$. eICIC scheme partitions the coordinated resources between the macro- and the picocells and allocates the interference-free resources of the almost blank subframes (ABSs) to the user equipment devices (UEs). The semistatic coordination technique of eICIC provides an advantage in intercell interference management but such a preserved resource management results in inefficient resource utilizations $[6,7]$.

For high-efficiency resource utilization, interference alignment (IA) was researched [8]. The concept of IA is to align the interference with the other transmitters into a reduced dimensional subspace at each receiver $[9,10]$. Then, IA enables an interference-free communication through the remaining signal subspaces for all the receivers and achieves half the multiplexing gain or the degrees of freedom (DoF).

Centralized IA [8] utilizes symbol extensions in any available domain. If the number of users of IA increases, the dimensions of the signal spaces induced by the symbol extensions increase and cause complex matrix operations. To avoid symbol extensions in the time or frequency domains, decentralized IA such as interference leakage minimization algorithm (ILMA) [11] was developed, enabling the alignment of the interference in a finite spatial domain. The deployment of the decentralized IA is restricted by the number of antennas and requires an additional complexity of at least tens of 
iterations for convergence [12]. Conventional IA schemes for heterogeneous networks, however, are restricted by the limited number of users and antenna configurations. For a large two-tier network, the number of participant users also cannot be increased. To overcome this problem, some researches proposed user selection schemes requiring additional system complexity that arranges the adequate number of the BS-UE pairs [13-16].

In practical heterogeneous networks, interference channels have not been clearly developed and some of the weak interference channels can be ignored. For example, picocells are deployed in a macrocell to cover the shadowing region and the interference from a macro BS to pico-UE is weak. However, if the location of the macro UE moves into the pico$\mathrm{BS}$, the macro UE is exposed to interference from the picoBSs. In this case, protection against the interference from a pico-BS to the macro UE is more important than that from a macro BS to the pico-UEs. Thus, the requirements of the precoding matrix designs for all the BSs in the conventional IA scheme can be relaxed.

Therefore, we propose a macrocell protection IA (MCPIA) method that utilizes finite dimensional orthogonal subspaces in the spatial domains without iterative computations. To align the interference of the macro UE from the picoBSs, signal subspaces of the macro UE with closed-forms are utilized and the interference rejection method is applied to the pico-UEs for reducing the interference from the macro BSs and the other pico-BSs. The proposed MCP-IA works well because the interference of the pico-UEs is small in practice. In addition, the proposed MCP-IA achieves diversity gain by selecting the subspace and the array gain by beamforming for the macro UE because the proposed MCP-IA constructs orthogonal subspaces of the macro UE between the signal and the interference subspaces. These two advantages are not obtained in the conventional IA. The DoF of the proposed MCP-IA is equal to or greater than that of the conventional IA. The proposed scheme is also effective to $5 \mathrm{G}$ systems since the carrier frequencies in $5 \mathrm{G}$ are considered as millimeter wave (mmWave), its indoor propagation and penetration losses are large, and it does not affect large interference to other indoor picocells [17-19].

To demonstrate the advantages of the proposed method, link level simulations are performed to compare the DoF and the capacity of the proposed MCP-IA with ILMA as the conventional IA, eICIC, and single-user multiple-input multiple-output (SU-MIMO) schemes; system level simulations are executed to provide the throughput of the pico- and the macrocells, for each scheme.

The rest of this paper is organized as follows. In Section 2, the system model is discussed. In Section 3, the design methods for precoding and the receiver matrices of the proposed scheme are described, and the achievable DoF and the optimality of the proposed MCP-IA are analyzed, and the complexity of the proposed MCP-IA is calculated. The numerical results are executed for the link level and the system level simulations in Section 4, and the conclusion follows in Section 5.

The notations used in this paper are defined as follows. Vectors and matrices are written in boldface with the matrices

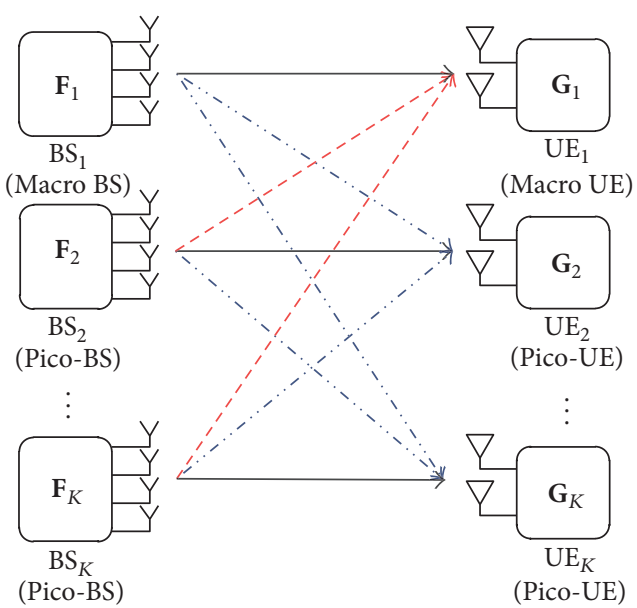

Figure 1: System model.

in capitals. All the vectors are column vectors. $\mathbf{A}^{H}$ denotes the conjugate transpose of $\mathbf{A} . \mathscr{C}(\mathbf{A})$ denotes the column space of $\mathbf{A} . \mathscr{R}(\mathbf{A})$ denotes the range space of $\mathbf{A} . \mathbf{A}^{\perp}$ and $\|\mathbf{A}\|_{F}$ denote the orthogonal complement and the Frobenius norm of $\mathbf{A}$, respectively. $\mathbf{I}_{n}$ denotes an identity matrix of size $n$. The random vector, $\mathbf{x} \sim \mathscr{C} \mathcal{N}(\boldsymbol{\mu}, \boldsymbol{\Sigma})$, denotes that $\mathbf{x}$ is drawn from a complex Gaussian distribution with a mean vector, $\boldsymbol{\mu}$, and a covariance matrix, $\boldsymbol{\Sigma}$. $v_{\min }^{d}(\mathbf{A})$ denotes a matrix whose columns are the eigenvectors of $\mathbf{A}$ corresponding to the $d$ smallest eigenvalues of $\mathbf{A} \cdot v_{\max }^{d}(\mathbf{A})$ denotes a matrix whose columns are the eigenvectors of $\mathbf{A}$ corresponding to the $d$ largest eigenvalues of $\mathbf{A}$. $\mathbb{E}[\cdot]$ denotes the expected value of a random variable.

\section{System Model}

For the heterogeneous networks, a single macrocell and $K-1$ picocells are considered, as in Figure 1. Each BS serves one UE per cell and the BSs are connected by backhaul links for exchanging a small amount of channel information. Let $\mathrm{BS}_{1}$ be a macrocell $\mathrm{BS}$ and $\mathrm{BS}_{k}$, where $k \neq 1$, be the picocell BSs. Each BS and UE are equipped with $M$ transmit antennas and $N$ receive antennas, respectively. A BS can transmit $d$ spatial streams that are bounded by $d \leq \min (M, N)$. Assume that all the MIMO channels experience slow fading and that precoding and data transmission are executed within coherence time. Each subcarrier of OFDM system has narrow band channel. The precoding matrix at each BS needs to be calculated for every channel realization.

The $k$ th BS, $\mathrm{BS}_{k}$, transmits $d$ symbols of the spatial symbol vector, $\mathbf{x}_{k} \in \mathbb{C}^{d}$, through $M$ transmit antennas with a linear precoder, $\mathbf{F}_{k} \in \mathbb{C}^{M \times d}$, where $\mathbf{x}_{k}$ satisfies the power constraint of $\mathbb{E}\left[\operatorname{tr}\left(\mathbf{x}_{k} \mathbf{x}_{k}^{H}\right)\right]=1$ and $\mathbf{F}_{k}$ satisfies $\operatorname{tr}\left(\mathbf{F}_{k} \mathbf{F}_{k}^{H}\right)=d$. Then, the received signal, $\mathrm{y}_{k}$, of the $k$ th user, $\mathrm{UE}_{k}$, is given by

$$
\mathbf{y}_{k}=\sqrt{P_{k k}} \mathbf{H}_{k k} \mathbf{F}_{k} \mathbf{x}_{k}+\sum_{j \neq k} \sqrt{P_{k j}} \mathbf{H}_{k j} \mathbf{F}_{j} \mathbf{x}_{j}+\mathbf{n}_{k},
$$

where $\mathbf{x}_{k}$ and $\mathbf{x}_{j}$ in (1) denote the desired signal and interference, respectively. $P_{k k}$ denotes the desired received signal 
power of $\mathrm{UE}_{k} \cdot \mathbf{H}_{k j}$ denotes a channel matrix from $\mathrm{BS}_{j}$ to $\mathrm{UE}_{k}$ with $\mathbf{H}_{k j} \in \mathbb{C}^{N \times M} . P_{k j}$ denotes the received interference power from $\mathrm{BS}_{j}$ to $\mathrm{UE}_{k}$, including the path loss and the shadowing. $\mathbf{n}_{k}$ denotes the additive complex white Gaussian noise at $\mathrm{UE}_{k}$ with a zero mean and a $\sigma_{n}^{2}$ variance, $\mathscr{C} \mathcal{N}\left(0, \sigma_{n}^{2} \mathbf{I}_{N}\right)$.

\section{Macrocell Protection Interference Alignment}

In this section, we propose a macrocell protection interference alignment in downlink two-tier heterogeneous networks. In two-tier heterogeneous networks, picocells are utilized to provide coverage extensions, shadow regions coverage, and offloading in a cell. If the picocells are randomly deployed, interference channel models for the heterogeneous networks may not be obtained and the conventional IA cannot be directly utilized for practical systems. If macro UEs are located near the picocells, the macro UEs are interfered by the picocell BSs, but pico-UEs are less interfered from the macro BSs because of their deployed locations. Thus, macro UEs need to be protected from the interference of the picocell BSs, while the pico-UEs may not require protection. The proposed MCP-IA considers practical cell environments and adopts IA concepts for the scenarios. The proposed algorithm is described in the following subsection.

3.1. Proposed MCP-IA. In order to suppress interference in practical heterogeneous networks, since the interference of the pico-UEs are small, full channel knowledge may not be required to align all the interference and IA can be developed for specific users who suffer from interference. In this paper, we develop the macrocell protection interference alignment wherein the cross-tier interference of the macro UE is suppressed by IA and the cross-tier interference of the picoUE is suppressed by receiver processing, based on the result of the IA. To mitigate the interference from the pico-BSs to the macro UE, the interference at the macro UE should be aligned. In addition, to achieve the DoF, all the interference to the macro UE should fall in the interference spaces of the macro UE. Then, the signal and interference spaces of the macro UE should be preserved and nulled, respectively.

For the first interference mitigation requirement, a perfect alignment of the cross-tier interference at the macro UE should be satisfied and is given by

$$
\mathscr{C}\left(\mathbf{H}_{12} \mathbf{F}_{2}\right)=\mathscr{C}\left(\mathbf{H}_{13} \mathbf{F}_{3}\right)=\cdots=\mathscr{C}\left(\mathbf{H}_{1 K} \mathbf{F}_{K}\right) .
$$

Equation (2) forms subspace equivalence based on the orthogonal complement and can be rewritten as the orthogonality of the desired signal and the interference subspaces of the macro UE, given by

$$
\begin{aligned}
\mathbf{G}_{1}^{H} \mathbf{H}_{1 k} \mathbf{F}_{k} & =\mathbf{0}, \quad \forall k \neq 1, \\
\operatorname{rank}\left(\mathbf{G}_{1}^{H} \mathbf{H}_{11} \mathbf{F}_{1}\right) & =d,
\end{aligned}
$$

where $\mathbf{G}_{1}$ denotes the interference suppression matrix of the macro UE. Since (3) is a system of bilinear equations, the system may have infinite solutions by initial values. If $\mathbf{G}_{1}$ is chosen as an appropriate value, however, the system can be easily solved without an iterative approach.

Let $\mathbf{u}_{i}$ be an arbitrary orthonormal basis to construct the orthogonal subspaces as follows:

$$
\begin{aligned}
& \mathcal{S}_{1}=\mathscr{C}\left(\left[\mathbf{u}_{1}, \mathbf{u}_{2}, \ldots, \mathbf{u}_{d}\right]\right), \\
& \mathscr{I}_{1}=\mathscr{C}\left(\left[\mathbf{u}_{d+1}, \mathbf{u}_{d+2}, \ldots, \mathbf{u}_{N}\right]\right),
\end{aligned}
$$

where $\delta_{1}$ and $\mathscr{I}_{1}$ denote the desired signal and the interference subspaces of the macro UE, respectively, and $\mathcal{S}_{1}, \mathscr{I}_{1} \neq$ $\emptyset$. The orthonormal basis, $\mathbf{u}_{i}$, can be obtained by several wellknown orthogonalization techniques such as Gram-Schmidt process, QR decomposition (QRD), or singular value decomposition (SVD).

3.2. Feasibility of the Proposed MCP-IA. The proposed MCPIA constructs the signal and interference subspaces arbitrarily, different from the conventional IA. Therefore, a feasibility condition for the proposed MCP-IA should be newly determined. The number of available spatial symbols between the macro BS and the pico-BS without cross-tier interference at the macro UE is investigated, and the following lemma is derived.

Lemma 1. For given $\mathbf{G}_{1}$, the number of spatial symbols, $d$, is bounded by

$$
d \leq \min \left(\frac{M}{2}, N\right)
$$

Proof. Let $d_{m}$ and $d_{p}$ be the number of spatial streams transmitted from a macro BS and a pico-BS, respectively. For given $\mathbf{G}_{1}$, the number of equations in (3) is $d_{m} d_{p}$. Using the uniqueness of the subspace in [9], the number of variables in (3) is given by $d_{p}\left(M-d_{p}\right)$. Therefore, $d_{m}$ and $d_{p}$ must satisfy the following condition:

$$
d_{p}\left(M-d_{p}\right) \leq d_{m} d_{p}
$$

Substituting $d_{m}=d_{p}=d$ in (7), $d$ can be obtained by

$$
d \leq \frac{M}{2} .
$$

As $d \leq \min (M, N)$, by the property of MIMO in Section 2, the number of spatial streams is bounded by

$$
d \leq \min \left(\frac{M}{2}, N\right)
$$

Lemma 1 shows that the feasibility of the proposed MCP-IA is not determined by the number of picocells but by the MIMO antenna configurations. Different from the conventional IA, if the number of transmit antennas is greater than that of the receive antennas, that is, $M \geq 2 N$, the proposed MCP-IA can achieve a greater DoF compared to the conventional IA. For example, in $4 \times 2 \mathrm{MIMO}$ systems, the conventional IA achieves DoF $=1$ only, even if it is feasible. The proposed IA, however, always attains $\mathrm{DoF}=2$, indicating that the proposed MCP-IA obtains larger spatial multiplexing gain without cross-tier interference. 
3.3. Precoding and Receiver Matrix Design of the Proposed $M C P-I A$. In this subsection, the precoding matrix of the transmitters and receivers for the proposed MCP-IA is derived based on the requirements of the previous subsection. The interference suppression matrix, $\mathbf{G}_{1}$, of the macro UE is directly derived when the orthogonal subspaces of the macro UE are chosen. Since $\mathbf{G}_{1}$ maps the macro BS's symbols into $\mathcal{S}_{1}$, that is, $\mathcal{S}_{1}=\mathscr{C}\left(\mathbf{G}_{1}\right), \mathbf{G}_{1}$ can be set as

$$
\mathbf{G}_{1}=\left[\mathbf{u}_{1}, \mathbf{u}_{2}, \ldots, \mathbf{u}_{d}\right]
$$

For the derivation of the precoding matrix, $\mathbf{F}_{1}, \mathbf{F}_{1}$ needs to be selected for the signal spaces given by (4). $\delta_{1}$ should be equivalent to a range space of $\mathbf{H}_{11} \mathbf{F}_{1}$; that is, $\mathcal{S}_{1} \subseteq \mathscr{R}\left(\mathbf{H}_{11} \mathbf{F}_{1}\right)$. Then, $\mathbf{F}_{1}$ is set as

$$
\mathbf{F}_{1}=\left[\mathbf{v}_{1}, \mathbf{v}_{2}, \ldots, \mathbf{v}_{d}\right]
$$

where $\mathbf{v}_{i}$ denotes the right singular vector of $\mathbf{G}_{1}^{H} \mathbf{H}_{11}$, corresponding to the $i$ th largest singular value.

If the DoF of the macro UE is greater than two, that is, $d \geq 2$, array processing and the transmit power allocation methods can be applied at the macro BS to improve the capacity performance. In the proposed MCP-IA scheme, the macro UE obtains an array gain and provides power allocations because $\mathbf{v}_{i}$ in $\mathbf{F}_{1}$ is calculated from $\mathbf{H}_{11}$ for the desired signal. Thus, the proposed MCP-IA can provide a greater signal-to-interference ratio (SIR) for the macro UE that is located in the edge of the macrocell and suffers from a low received signal power and cross-tier interference. Note that the conventional IA is not designed for maximizing the capacity and the array gain of the desired signal subspace.

The precoding matrix of the picocell BSs, $\mathbf{F}_{k}, \forall k \neq 1$, can be calculated by (3) and is a homogeneous system of linear equations. If $\mathbf{F}_{k}=\mathbf{0}$, the solution set of the system is trivial and the picocell BSs do not transmit. For a nonnontrivial solution of (3), the nonzero null space of $\mathbf{G}_{1}^{H} \mathbf{H}_{1 k}$ is the solution. For example, the nonzero null space of $\mathbf{G}_{1}^{H} \mathbf{H}_{1 k}$ can be calculated by the selection of the right singular vector of the SVD of $\mathbf{G}_{1}^{H} \mathbf{H}_{1 k}$ with vanishing singular values. As $\mathbf{G}_{1}^{H} \mathbf{H}_{1 k} \in \mathbb{C}^{d \times M}$ and $d \leq \min (M / 2, N), \mathbf{G}_{1}^{H} \mathbf{H}_{1 k}$ has $M-$ $d$ zero singular values. Then, the precoding matrix, $\mathbf{F}_{k}$, of picocell BSs, $\forall k \neq 1$, is obtained as

$$
\mathbf{F}_{k}=\left[\overline{\mathbf{v}}_{M-d+1}^{k}, \overline{\mathbf{v}}_{M-d+2}^{k}, \ldots, \overline{\mathbf{v}}_{M}^{k}\right], \quad \forall k \neq 1
$$

where $\overline{\mathbf{v}}_{i}^{k}$ denotes the right singular vector of $\mathbf{G}_{1}^{H} \mathbf{H}_{1 k}$ corresponding to the $i$ th largest singular value. Thus, $\mathbf{G}_{1}$ and all $\mathbf{F}_{k}$ satisfy the requirements of (3) and the proposed MCP-IA provides the DoF to the macro UE and suppresses the crosstier interference of the macro UE from the other picocell BSs.

Since one of the main goals of the proposed MCP-IA is to protect the macro UE from the cross-tier interference, the achievable DoF of the proposed MCP-IA for the macro UE is important. Thus, the DoF of the proposed MCP-IA is derived by the following lemma.
Lemma 2. The achievable DoF of the macro UE are given as follows:

$$
\lim _{\rho \rightarrow \infty} \frac{C_{M}(\rho)}{\log _{2}(\rho)}=d,
$$

where $C_{M}(\rho)$ and $\rho$ denote the capacities of the macro UE and signal-to-noise ratio (SNR), respectively.

Proof. See Appendix A.

Let us consider the interference suppression matrix of the pico-UE. The interference of the pico-UEs from the macro BSs is not significant and the interference among the picocells is very small. In this scenario, the conventional strict IA need not be applied for designing $\mathbf{G}_{k}$. In the proposed MCP-IA, the precoding matrix, $\mathbf{F}_{k}, \forall k \neq 1$, of the picocell $\mathrm{BS}$ is aligned to the null spaces of the macro UE but not for other pico-UEs. The interference of the pico-UEs exist and are accumulated from the other picocell BSs and the macrocell BSs. Therefore, an interference mitigation method for the pico-UEs needs to be developed. The distribution of the accumulated interference can be modeled as Gaussian by the central limit theorem. In this paper, an MMSE-IRC (minimum mean squared error interference rejection combining) receiver is adopted to mitigate the interference and is given by

$$
\mathbf{G}_{k}=\frac{1}{\beta_{k}}\left(\mathbf{Q}_{k}+\sigma_{n}^{2} \mathbf{I}_{N}\right)^{-1} \mathbf{H}_{k k} \mathbf{F}_{k}, \quad \forall k \neq 1,
$$

where $\beta_{k}$ denotes the normalization factor satisfying $\left\|\mathbf{G}_{k}\right\|_{F}^{2}=$ d. $\mathbf{Q}_{k}$ denotes the interference covariance matrix of the $k$ th pico-UE and is given by

$$
\mathbf{Q}_{k}=\sum_{j \in \mathscr{B}_{k}} \frac{P_{k j}}{d} \mathbf{H}_{k j} \mathbf{F}_{j} \mathbf{F}_{j}^{H} \mathbf{H}_{k j}^{H},
$$

where $\mathscr{B}_{k}$ denotes the interference of the $k$ th pico-UE from other BSs. $\mathscr{B}_{k}$ depends on the cross-correlation among the picocells. If the channels between the pico-UEs and the other pico-BSs are correlated or the channel gains are very small, the rank of $\mathbf{Q}_{k}$ is less than or equal to $(N-d)$ and the $k$ th pico-UE has a $d$-dimensional interference-free subspace; that is, the pico-UE preserves the DoF without a complex interference alignment. This scenario is common in practical heterogeneous networks [20]. If many picocells are colocated and the interference from the other pico-BSs is strong and uncorrelated, that is, $N-d<\operatorname{rank}\left(\mathbf{Q}_{k}\right) \leq N$, the picoUEs of the proposed method may not receive any signals from their BSs. In practice, however, this does not happen frequently. In the following Numerical Results section, the system level simulation results show that the proposed MCPIA demonstrates a greater system throughput compared to the conventional IA.

For the link capacity maximization of the macro UE, the conventional IA schemes do not maximize the capacity since the precoding matrices of them are designed for achieving the DoF. The macro BS of the proposed MCP-IA, however, obtains the maximum link capacity with the optimal precoding matrix, which is important at the low SNR regime 
of the cellular environment. The optimal precoder design of pico-BSs that operates in the large SNR regime may not be important because their capacities are close to the optimal capacity. The optimality of the macro BS of the proposed MCP-IA is shown in Lemma 3.

Lemma 3. The conditions finding the optimal precoding matrix $\mathbf{F}_{1}$ are given as follows:

(1) For $M<2 N, \mathbf{F}_{1}$ is optimal if the columns of $\mathbf{G}_{1}$ are the left singular vectors of $\mathbf{H}_{11}$.

(2) For $M \geq 2 N, \mathbf{F}_{1}$ is always optimal regardless of $\mathbf{G}_{1}$.

Proof. See Appendix B.

3.4. Complexity of MCP-IA. One of the benefits of the proposed MCP-IA is the low computational complexity compared with that of the conventional IA (ILMA). The comparison is performed by the total number of floating point operations (FLOPs).

Table 1 exhibits the number of FLOPs for the proposed MCP-IA and ILMA. In Table $1, \Sigma$ and $V$ and $U$ denote singular values and right and left singular matrices, respectively. $\left|\mathscr{B}_{k}\right|$ denotes the number of interferers of the $k$ th pico-UE from other BSs. $\overrightarrow{\mathbf{Q}_{k}}$ denotes an interference covariance matrix of reverse link in ILMA. $L$ denotes the number of QR iterations for eigenvalue decomposition. $L$ is set as 5 so that the mean square error of the eigenvector is lower than $10^{-4}$. The conventional ILMA requires more than 70 iterations to converge [21]. As seen in Table 1, the significant computational saving of the proposed MCP-IA for $4 \times 2$ antenna configuration is obtained compared with the conventional ILMA.

In $5 \mathrm{G}$ mmWave small cell environments, interference among picocells is reduced and $\left|\mathscr{B}_{k}\right|$ decreases, too. Thus, the proposed MCP-IA is implemented with lower complexity.

\section{Numerical Results}

We evaluate the link and the system level performance of the proposed MCP-IA compared to that of the conventional IA, for which interference leakage minimization algorithm (ILMA) is used, and other interference suppression methods. First, in link level simulations, an isolated heterogeneous network with fixed SIRs for the cross-tier and cotier interference is assumed. The capacity of the UEs versus the SNR is evaluated and the achievability of the DoF at the macro UE is verified. The effects of the channel estimation errors are tested and the optimality of the proposed MCP-IA is evaluated. Next, in the system level simulation, the throughputs versus the number of picocells of the proposed MCP-IA under multiple macrocells are executed. The system level simulation parameters are summarized in Table 2.

4.1. Link Level Performance. The link level simulation shows the capacities of the UEs versus the SNR and exhibits the DoF of the proposed MCP-IA, ILMA, and SU-MIMO for heterogeneous networks. The number of picocells varies from one to four for one macrocell. $2 \times 2$ antenna configuration and $4 \times 2$ antenna configuration are considered. A macrocell and a picocell contain one UE per cell. The received signal power of all the UEs varies and the noise variance, $\sigma_{n}^{2}$, at the UEs is set to be one. For the interference, the average SIR for the cross-tier is set to be $3 \mathrm{~dB}$ and the average SIR for the cotier interference is set to be $20 \mathrm{~dB}$. SU-MIMO is compared to observe interference effects from other BSs. SU-MIMO is not designed to suppress the interference but treats the interference as additive noise.

For link capacity comparison, the capacity equation including the other cell interference, precoding and interference suppression matrices, is defined as follows:

$$
C_{k}=\log _{2} \operatorname{det}\left(\mathbf{I}_{N}+\frac{\left(P_{k k} / d\right) \mathbf{G}_{k}^{\mathrm{H}} \mathbf{H}_{k k} \mathbf{F}_{k} \mathbf{F}_{k}^{\mathrm{H}} \mathbf{H}_{k k}^{\mathrm{H}} \mathbf{G}_{k}}{\mathbf{G}_{k}^{\mathrm{H}}\left(\mathbf{Q}_{k}+\sigma_{n}^{2} \mathbf{I}_{N}\right) \mathbf{G}_{k}}\right) .
$$

In Figures 2, 3, and 4, $C_{\mathrm{MU}}=C_{1}$ and $C_{\mathrm{PU}}=C_{k}, \forall k \neq$ 1 , denote the capacity of the macro UE and the pico-UE, respectively.

For a $2 \times 2$ MIMO system, SU-MIMO utilizes dual-layer spatial multiplexing, while the proposed MCP-IA and ILMA do not. For a $4 \times 2$ case, as the DoF of the proposed MCP-IA is two by Lemma 1 and the DoF of ILMA is one by [8], the proposed MCP-IA and SU-MIMO utilize dual-layer spatial multiplexing, while ILMA does not.

The capacities versus the SNR of the UEs for one picocell, two picocells, and four picocells are displayed in Figures 2, 3 , and 4, respectively. The diamond-solid line denotes the capacity of the macro UE of the proposed MCP-IA, the circlesolid line denotes that of the pico-UE of the proposed MCPIA, the inverse triangle-solid line denotes that of the macro UE of ILMA, the triangle-solid line denotes that of the picoUE of ILMA, the plus-solid line denotes that of the macro UE of SU-MIMO, the $x$-solid line denotes that of the pico-UE of SU-MIMO, and dashed line denotes the DoF of the IA.

As shown in Figures 2(a) and 2(b), the capacity of ILMA and the proposed scheme increase linearly. The capacity of SU-MIMO does not increase linearly because SU-MIMO is not designed to suppress the interference between the picocell and the macrocell. The capacity of the macro UE of the proposed MCP-IA is greater than that of the other methods. This is because the proposed MCP-IA has diversity and array gains compared to ILMA. The diversity gain is obtained by the selection of the signal space among the orthogonal spaces of macro UE. The array gain is attained by the fact that the precoder is designed for a link between macro BS and macro UE. Recall that the precoders of ILMA are not designed to increase the received signal power, but only to align the interferences of all BSs. In Figure 2(a), for a $2 \times 2$ MIMO antenna case, pico-UE of the proposed MCP-IA and ILMA attains the same DoF, but in Figure 2(b), for a $4 \times 2 \mathrm{MIMO}$ antenna case, the proposed MCP-IA achieves a double DoF and a more than a double capacity compared to ILMA. This is because Lemma 1 tells that the proposed MCP-IA with $M \geq 2 N$, that is, $M=4$ and $N=2$, always achieves $d=N$ DoF for the macro UE, while ILMA has $d \leq N / 2$ DoF by (1) in [11] even if it is feasible. In practice, the number of antennas at the BS is greater than that of the UEs, and the proposed MCP-IA is a more effective scheme than ILMA for obtaining a greater capacity. 


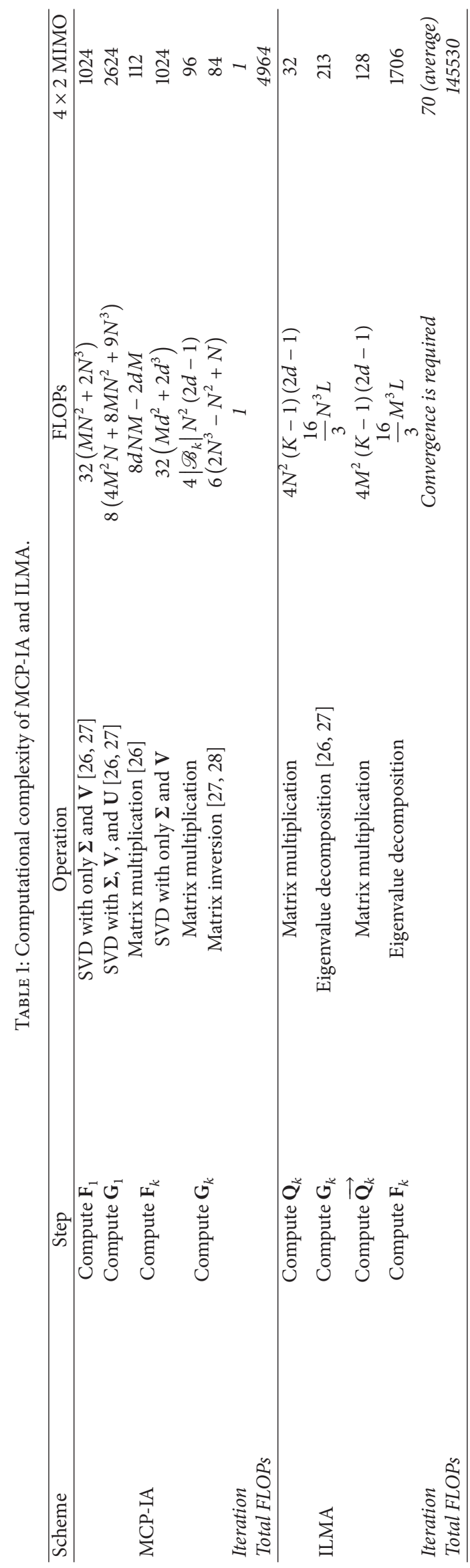




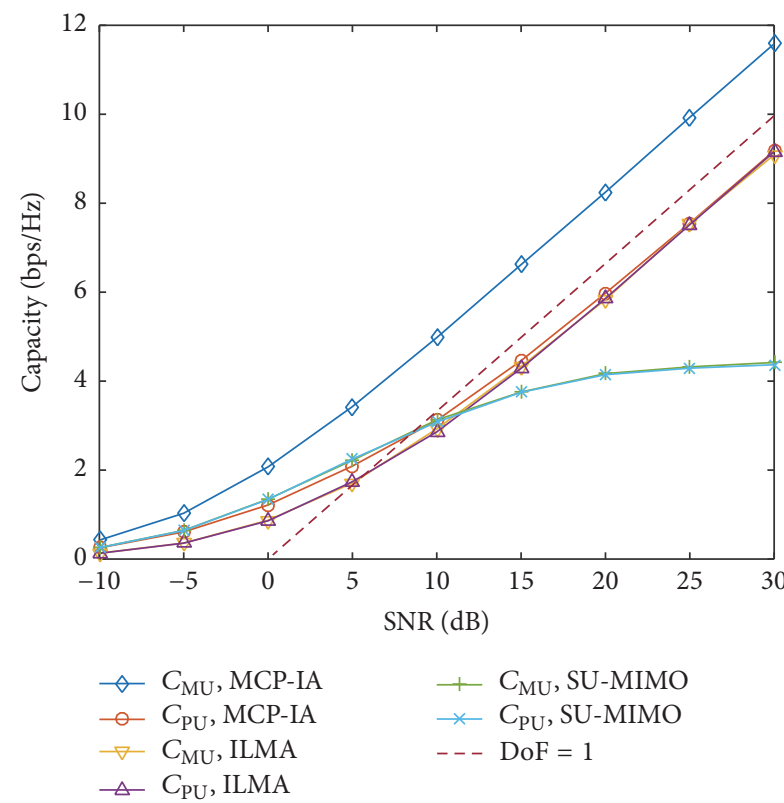

(a) Link capacity of $2 \times 2 \mathrm{MIMO}$ antenna configuration

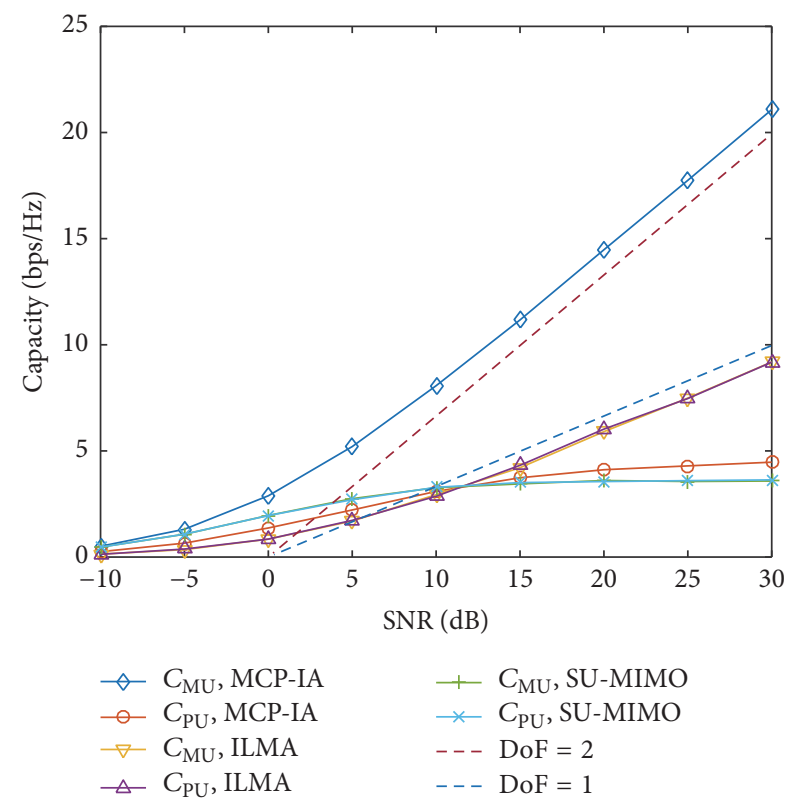

(b) Link capacity of $4 \times 2 \mathrm{MIMO}$ antenna configuration

FIGURE 2: Link capacity of UE with one picocell.

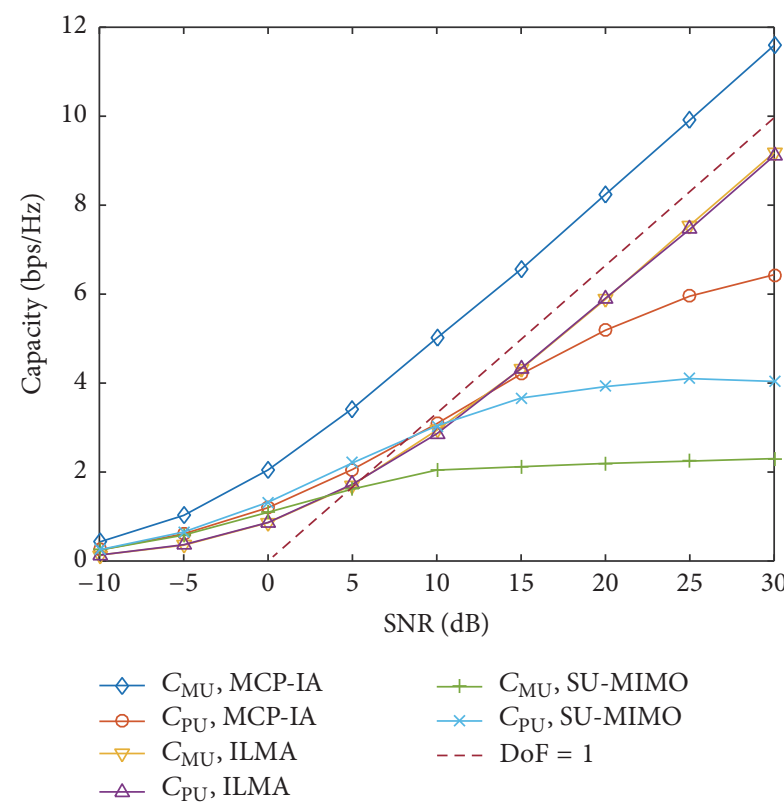

(a) $2 \times 2$ MIMO antenna configuration

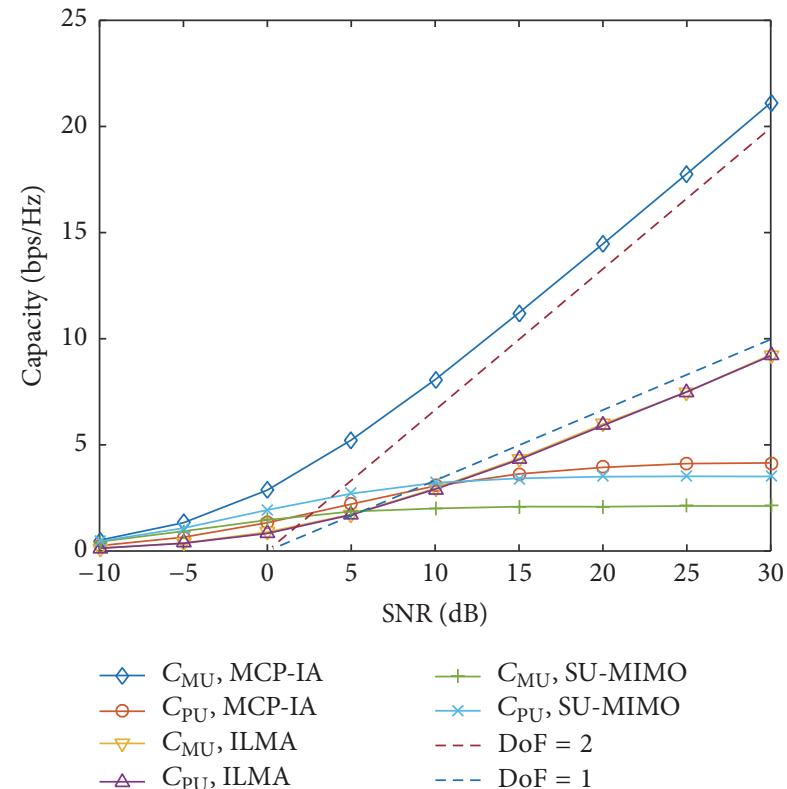

(b) $4 \times 2$ MIMO antenna configuration

FIGURE 3: Link capacity of UE with two picocells.

For a two-picocell scenario in Figures 3(a) and 3(b), ILMA still has a feasible solution and the capacities of the macro UE are similar to the one-picocell scenarios. The proposed MCP-IA shows a capacity loss in the pico-UE for an SNR region larger than $15 \mathrm{~dB}$ due to the interferences from the other pico-BSs. In practical environments, as the pico-UE is located closer to the pico-BS than the other pico-BSs, the pico-UE always preserves a greater capacity and the capacity loss is negligible. For a $4 \times 2$ case, the proposed MCP-IA obtains a multiplexing gain that results in a larger capacity than ILMA. This is also seen in the system level simulation.

In Figures 4(a) and 4(b), the capacities versus the SNR of the UEs are displayed for four pico-BSs. In Figure 4(a), the precoder solution of the ILMA for $2 \times 2$ antenna configuration is not available. The macro UE of ILMA with $2 \times 2$ MIMO systems shows a capacity loss in an SNR range 


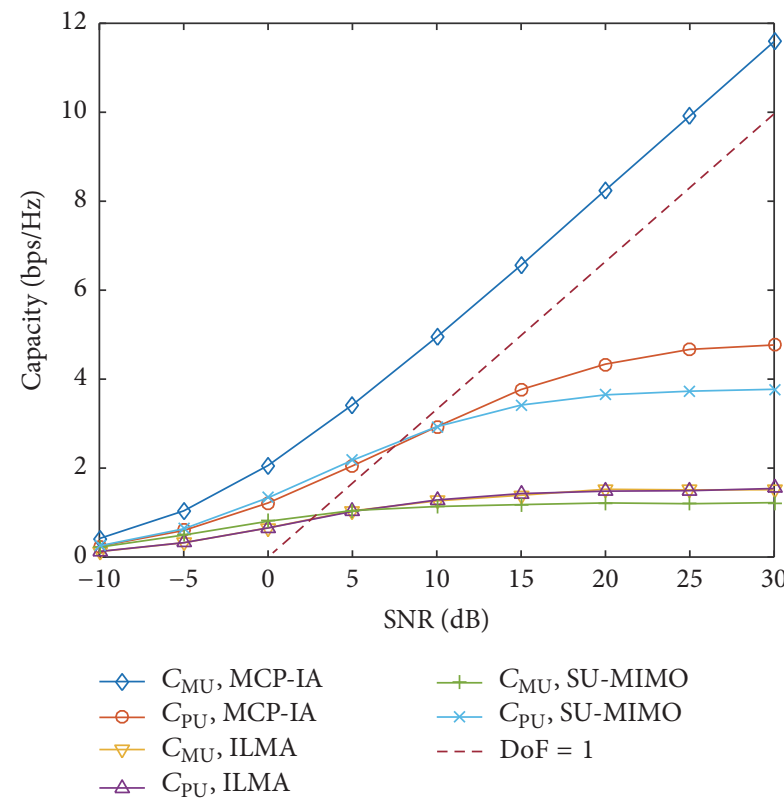

(a) $2 \times 2$ MIMO antenna configuration

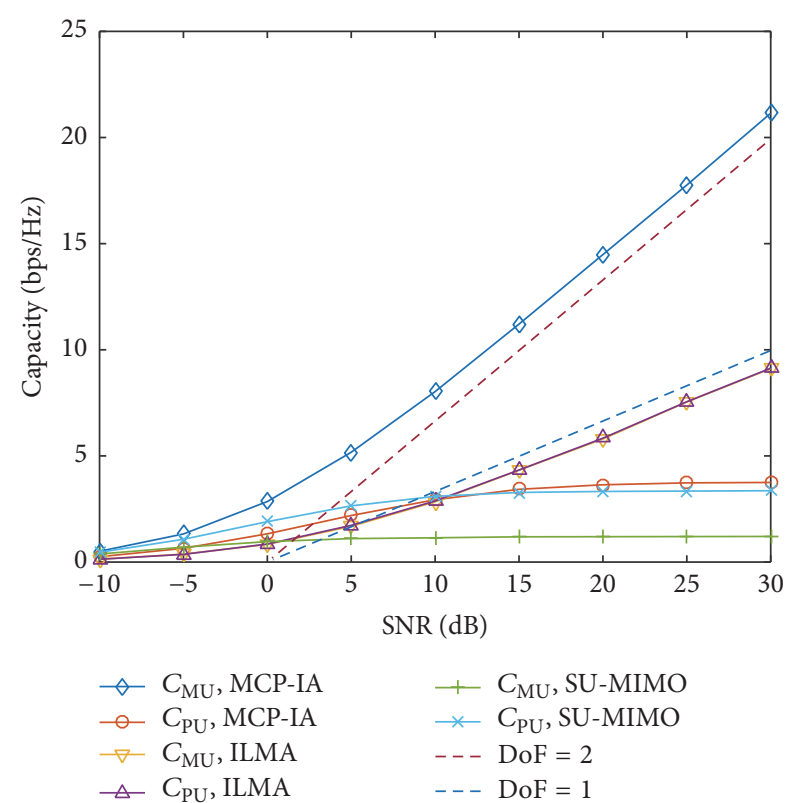

(b) $4 \times 2$ MIMO antenna configuration

FIGURE 4: Link capacity of UE with four picocells.

TABLE 2: Simulation parameters.

\begin{tabular}{ll}
\hline Parameter & Assumption \\
\hline Macrocell layout & $\begin{array}{l}19 \text { hexagonal cells, } 3 \text { sectors per } \\
\text { cell }\end{array}$ \\
$\begin{array}{l}\text { Inter-MBS distance } \\
\text { Picocell coverage }\end{array}$ & $200 \mathrm{~m}$ \\
Carrier frequency/bandwidth & $2 \mathrm{GHz} / 20 \mathrm{MHz}$ \\
MBS transmission power & $46 \mathrm{dBm}$ \\
PBS transmission power & $23 \mathrm{dBm}$ \\
Path loss from the MBS to the & $128.1+37.6 \log _{10} R[\mathrm{~dB}], R$ in $\mathrm{km}$ \\
UE & \\
Path loss from the PBS to the & $140.7+36.7 \log _{10} R[\mathrm{~dB}], R$ in $\mathrm{km}$ \\
UE & $A(\theta)=-\min \left[12\left(\theta / \theta_{3 \mathrm{~dB}}\right)^{2}, A_{m}\right]$ \\
MBS antenna pattern & Omnidirectional \\
PBS antenna pattern & ITU-R M.1225 Ped. A \\
Channel model & $8 \mathrm{~dB}$ \\
Shadowing standard deviation & $20 \mathrm{~dB}$ \\
Penetration loss & $9 \mathrm{~dB}$ \\
Noise figure & $-174 \mathrm{dBm} / \mathrm{Hz}$ \\
Noise power spectral density & Full buffer \\
Traffic model &
\end{tabular}

greater than $10 \mathrm{~dB}$, as does the pico-UE. If the number of picocells increases, ILMA is not feasible and cannot achieve the DoF [9]. The macro UE capacity of the proposed MCPIA, however, increases linearly even though the number of picocells increases. The pico-UE capacities of the proposed MCP-IA and ILMA do not linearly increase. This is because the proposed MCP-IA does not align all the interferences

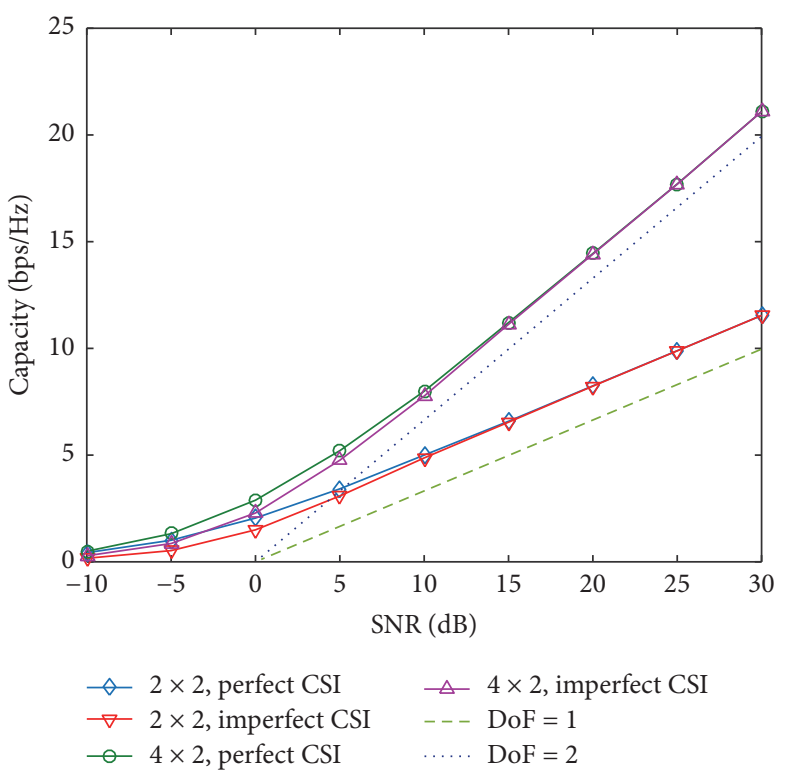

FIGURE 5: Link capacity of the macro UE with/without channel estimation error.

of the UEs but suppresses the interference of the macro UE from the pico-BSs, as in Figures 2 and 3. Recall that in general heterogeneous networks most picocells are located to cover the shadowing region of the macrocell and are not considerably interfered by the macro BSs. In SU-MIMO case, the capacity is saturated by the cross-tier interferences between the macrocell and the picocells, and it shows the worst capacity result among all the cases. In Figure 4(b) with $4 \times 2$ antenna configuration, ILMA is feasible and achieves 


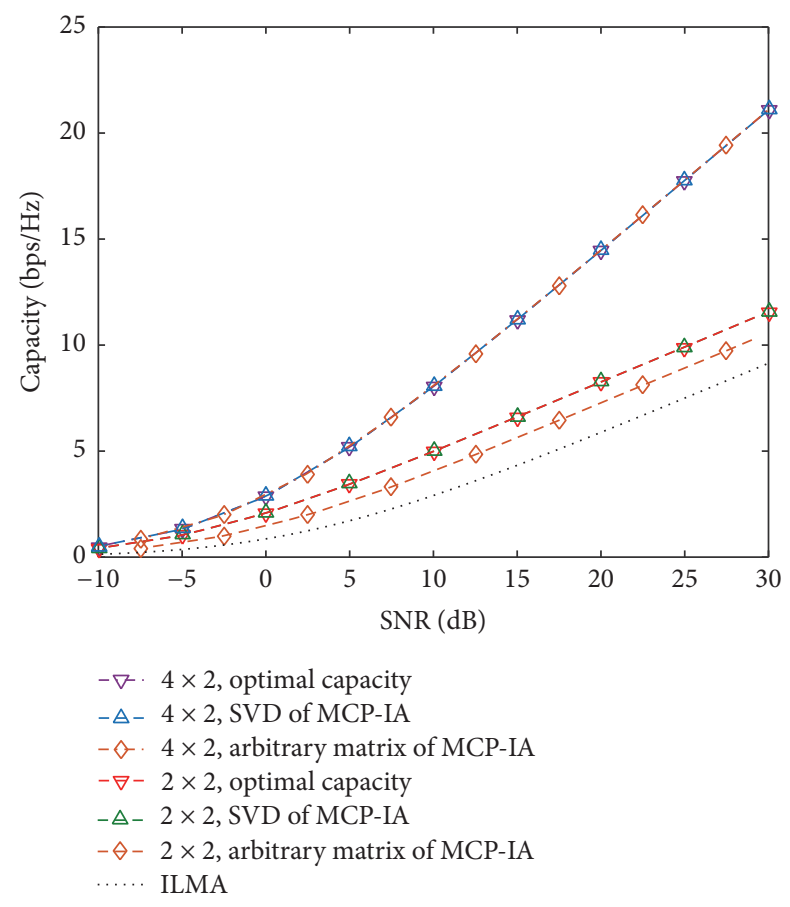

FIGURE 6: Optimality of capacity comparison for the macro UE.

the DoF for the macro UE and pico-UE. However, as with Figure 3, the DoF and the capacity of the proposed MCPIA are double those of ILMA and the proposed MCP-IA has additional array and diversity gains.

Figure 5 illustrates the capacity performance of the macro UE of the proposed MCP-IA with the channel estimation error. Least square (LS) is utilized for the channel estimation. In Figure 5, "perfect CSI" and "imperfect CSI" denote the capacity performances without and with the channel estimation error, respectively. The capacity loss by the channel estimation error is calculated in [22, 23]. Even though the channel estimation error occurs, the proposed MCP-IA always achieves the DoF and exhibits the small channel capacity loss which is within the errors derived in $[22,23]$. Thus, the proposed MCP-IA is robust to the channel estimation errors.

Figure 6 exhibits capacities by the optimum capacity and SVD with the proposed MCP-IA and arbitrary matrix with the proposed MCP-IA and ILMA. In Figure 6, dashed line and dash-dotted line denote the capacities of $2 \times 2$ and $4 \times 2$ MIMO antenna configuration, respectively, and dotted line denotes the capacity of ILMA. Let the $\operatorname{DoF} d$ be $\min (M / 2, N)$. The SVD of MCP-IA and the arbitrary matrix of MCPIA are obtained in (5). In case of $2 \times 2 \mathrm{MIMO}$, as seen in Lemma 3, the SVD of MCP-IA demonstrates the same capacity performance as the optimum capacity, and the arbitrary matrix of MCP-IA exhibits smaller capacity. For $4 \times 2$ MIMO configuration, all of the proposed MCP-IAs attain the optimal capacity. ILMA, however, has lower capacity than the proposed MCP-IAs. This is because ILMA is not designed for maximizing the capacity but for aligning the interference to achieve the DoF. Therefore, the proposed
MCP-IA demonstrates better capacity performance than ILMA.

The proposed MCP-IA is designed to protect the macro UEs from the pico-BSs and maximize the capacity. The signal subspace of the macro UE is selected to maximize the capacity and the interference subspace is chosen to align the crosstier interference. Then, the macro UE achieves the DoF and the optimal capacity. As seen in Figures 2(b), 3(b), and 4(b), for asymmetric antenna configuration such as $M \geq 2 N$, the macro UE of the proposed MCP-IA always guarantees $d=N$ DoF by Lemma 1, while ILMA does $d \leq N / 2$ DoF even if ILMA is feasible. All the interference suppression schemes have the DoF loss compared to the available DoF of $\min (M, N)$ of the MIMO multiplexing scheme because their DoF are utilized to cancel the interference. The proposed MCP-IA achieves $\min (M / 2, N)$ DoF that is greater than $\min (M, N) / 2$ DoF of ILMA when the number of antennas of the BS is greater than that of the UEs. The $M \geq 2 N$ antenna configuration is common in practical systems and the proposed MCP-IA is more applicable than ILMA.

In the next subsection, the system level simulation is performed to verify the throughputs in randomly deployed picocells and in multiple macrocells.

4.2. System Level Performance. In the system level simulation, we measure the throughputs of multiple macrocells with the other cell interference. Since the solutions of the conventional IA schemes such as ILMA with a number of picocells do not exist, eICIC is utilized for comparison. Thus, eICIC, SUMIMO, and the proposed MCP-IA schemes are evaluated.

We consider that the macro UEs and the pico-UEs move in their cells randomly and that the interference among them occurs by their moving locations. 19 hexagonal macrocells are deployed, each macrocell consists of three sectors, and the number of picocells in a macrocell varies from 2 to 20 . The number of macro UEs in a macrocell and pico-UEs in a picocell is set to 20 and 5, respectively. For simplicity, we measure the throughput of the macro UEs and picoUEs in a center cell. Then, the macro UE and pico-UEs are interfered from the other 18 macrocells. The locations of the macro UE are sometimes close to the picocells resulting in strong interference scenarios. $2 \times 2$ antenna configuration and $4 \times 2$ antenna configuration are tested. SU-MIMO and the proposed MCP-IA utilize the same spatial symbol dimensions as in the previous link level simulations, and eICIC utilizes dual-layer spatial multiplexing. The other communication parameters for the system level simulations are listed in Table 2. The system level simulations in this paper conform to the evaluation methodology of 3GPP [24].

Figures 7(a) and 7(b) show the throughput of macro UE versus the number of picocells from 2 to 20 for the proposed MCP-IA, eICIC, and SU-MIMO schemes under $2 \times 2$ antenna configuration. The throughput denotes an average value of 20 macro UEs for a macrocell. Inverse triangles, triangles, and circles denote the throughputs of the proposed MCP-IA, eICIC, and SU-MIMO schemes, respectively. In Figure 7(a), the proposed MCP-IA demonstrates the maximum throughput performance among the three schemes. Even though the number of picocells increases, the proposed MCP-IA 


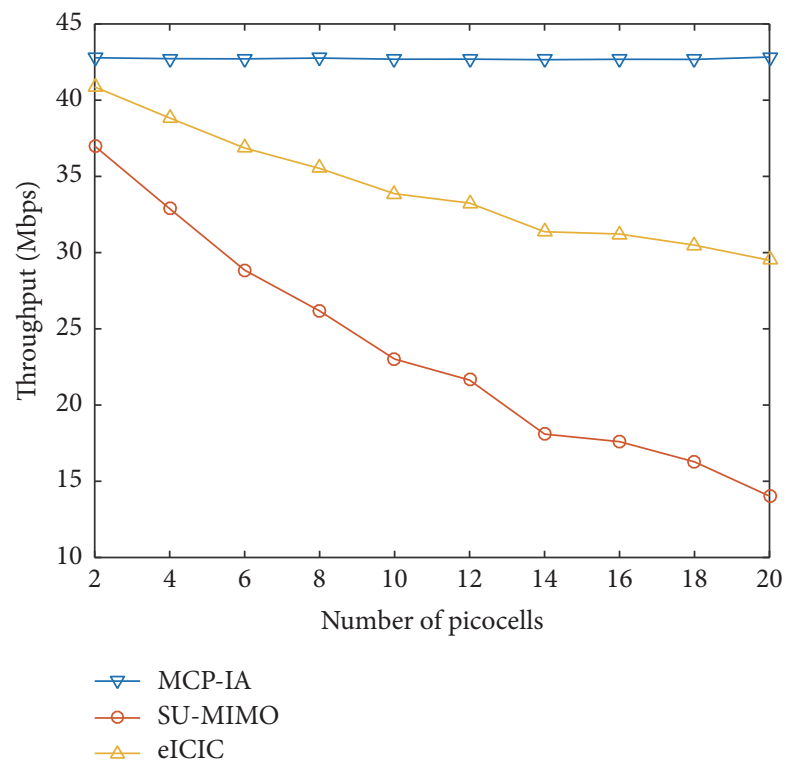

(a) Macrocell throughput

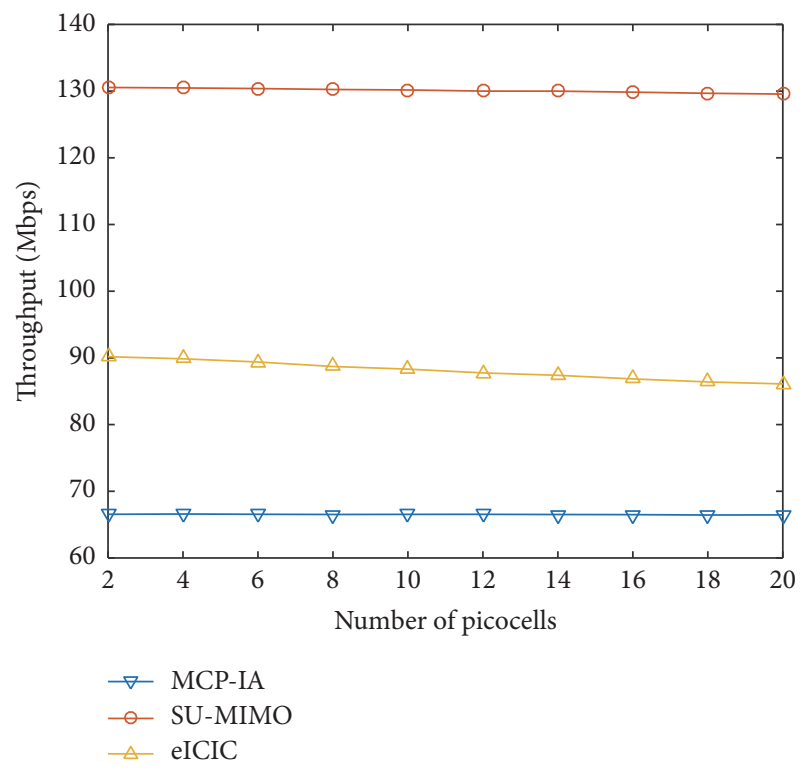

(b) Per-picocell throughput

FIGURE 7: System level performance of the cell throughput corresponding to the number of picocells with $2 \times 2$ antenna configuration.

maintains the DoF and the maximum throughput of the macro UE, while the throughputs of the other methods decrease. This indicates that the proposed MCP-IA protects against interferences from the pico-BSs completely, even though the number of picocells increases.

Figure 7(b) displays the throughputs of the picocells versus the number of picocells of the proposed MCP-IA, eICIC, and SU-MIMO. The throughput of the pico-UEs denotes the average value of five pico-UEs in a picocell. The distances between the pico-BS and the pico-UEs are relatively closer than those between the macro BS and the picoUEs. The cross-tier interference of the pico-UEs is relatively small because of path loss and shadowing, and the cotier interference among the picocells is also small because the density of the picocells in a macrocell is sparse. Therefore, the SNR range of the picocell is greater than that of the macrocell and the average throughput of the picocell is also greater than that of the macrocell. In Figure 7(b), a constant throughput of the proposed MCP-IA demonstrates that the proposed MCP-IA is not affected by the number of picocells and suppresses the interference successfully. The throughput of eICIC and SU-MIMO decreases as the number of picocells increases due to interferences from the other picocells. This is because SU-MIMO and eICIC do not suppress interference and the proposed MCP-IA does. Then, SU-MIMO and eICIC can utilize two spatial streams, and the proposed MCP-IA has one spatial stream with half of the throughput of SUMIMO. In practice, however, the number of antennas of the BSs is larger than that of UEs, for example, $4 \times 2$. In this case, the proposed MCP-IA also has two spatial streams and similar throughput of SU-MIMO. This result is shown in Figure 8.

Figure 8 depicts the throughputs versus the number of picocells for $4 \times 2$ antenna configuration. The throughput of the macrocell in Figure 8(a) shows similar results of the $2 \times 2$ simulations in Figure 7(a) but increases owing to the large number of transmit antennas. The macrocell throughput of the proposed MCP-IA also demonstrates the largest value among the three schemes and is not affected by the number of picocells. Although the proposed MCP-IA utilizes two spatial streams, the throughput of the macro BS may not be doubled because the SNR regime of the macro UE is low. This result can be seen in Figure 6. At the low SNR region in Figure 6, the capacity of macro UE with the $4 \times 2$ configuration is not doubled compared to that of $2 \times 2$. In Figure 8(b), the picocell throughput of the proposed MCP-IA outperforms that of eICIC and approaches that of SU-MIMO. This is because the proposed MCP-IA utilizes two spatial streams and eICIC inefficiently manages its resources due to the ABS subframes. The proposed MCP-IA precoders of the pico-BSs are not designed to improve the capacity of pico-UEs but to align cross-tier interference. The throughput of the proposed MCP-IA is little bit lower than SU-MIMO. However, since the SNR region of the pico-UEs is large, the throughput of the pico-UEs approaches that of SU-MIMO. This throughput gap between the proposed MCP-IA and SU-MIMO is about $3 \%$. Therefore, the proposed MCP-IA provides a better total throughput than eICIC and SU-MIMO in heterogeneous networks.

In $5 \mathrm{G}$ systems, larger carrier frequency such as mmWave will be utilized, and many picocells are deployed in indoor environments, and all equipment has MIMO to increase capacity. In the scenarios, cotier interference is relatively small and cross-tier interference is large. This provides relaxed design rules for IA and the proposed MCP-IA is developed. Thus, the proposed MCP-IA is applicable to reduce cross-tier interference of heterogeneous networks in the $5 \mathrm{G}$ system. 


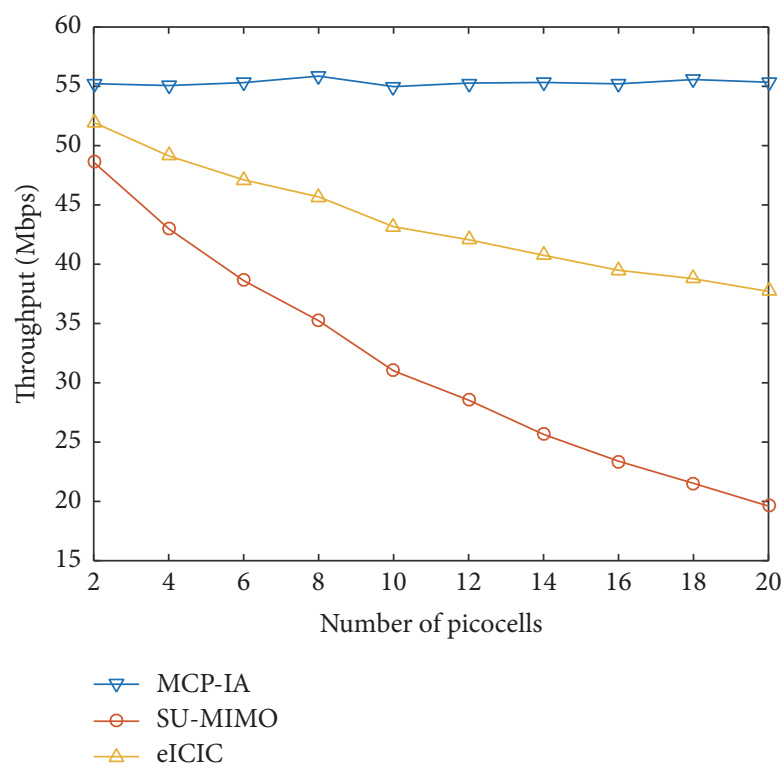

(a) Macrocell throughput

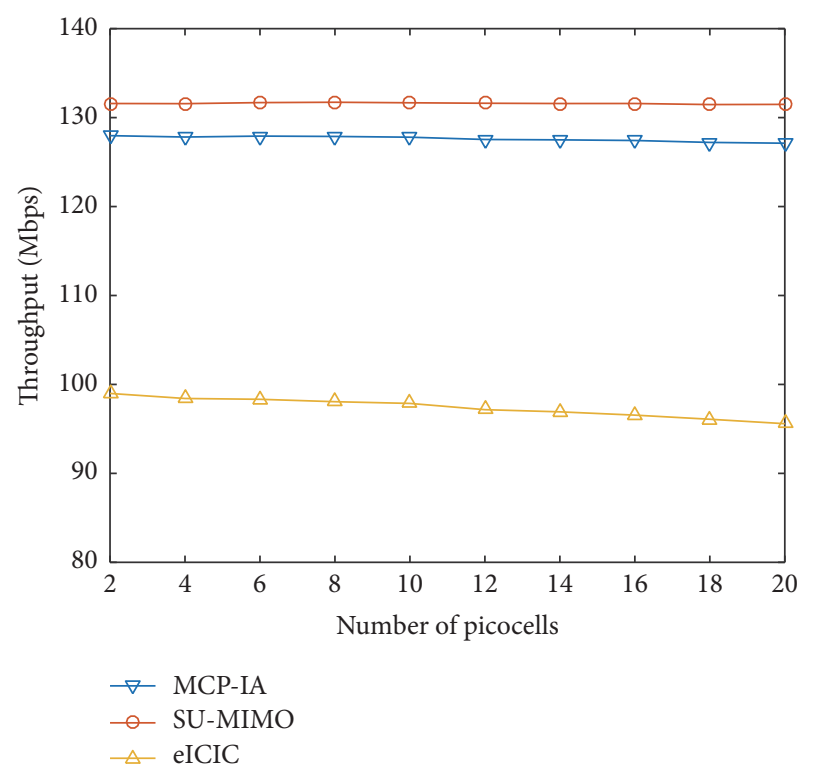

(b) Per-picocell throughput

FIGURE 8: System level performance of the cell throughput corresponding to the number of picocells for $4 \times 2$ antenna configuration.

\section{Conclusion}

This paper proposes a MCP-IA method for two-tier MIMO downlink heterogeneous networks. The proposed MCP-IA utilizes the concept of IA for the macro UE for protection against cross-tier interference and achieves the same DoF as that of the conventional IA for the macro UE. The picoUE suppresses the interference from the macro BS using MMSE-IRC. The proposed MCP-IA provides additional array and diversity gains for the macro UE compared to the conventional IA. The array gain of the proposed method is achieved by beamforming precoder for the macro UE and the diversity gain is attained by selecting good signal spaces among the available spaces. The proposed MCP-IA calculates the precoding matrices of the BSs with a closed form. The DoF of the proposed MCP-IA is equal to or greater than the conventional IA and is derived theoretically. The link level simulation results show that the proposed MCP-IA achieves the DoF and the additional array and diversity gains. The system level simulation demonstrates that the proposed MCP-IA suppresses the interference of the macro UE completely and that the pico-UEs maintain the throughput even under the interferences of a large number of picocells. For $4 \times 2$ antenna configuration, the system level simulation demonstrates that the proposed MCP-IA obtains the additional multiplexing gain.

\section{Appendix}

\section{A. Proof of Lemma 2}

The interference-aware ergodic capacity of the macro UE can be written as [25]

$$
\begin{aligned}
& C_{M}(\rho) \\
& \quad=\mathbb{E}\left[\log _{2} \operatorname{det}\left\{\mathbf{I}_{d}+\frac{\rho}{d} \frac{\mathbf{G}_{1}^{H} \mathbf{H}_{11} \mathbf{F}_{1} \mathbf{F}_{1}^{H} \mathbf{H}_{11}^{H} \mathbf{G}_{1}}{\mathbf{G}_{1}^{H}\left(\mathbf{Q}_{1}+\mathbf{I}_{N}\right)^{-1} \mathbf{G}_{1}}\right\}\right],
\end{aligned}
$$

where

$$
\mathbf{Q}_{1}=\sum_{j \in \mathscr{B}_{1}} \frac{P_{1 k}}{d} \mathbf{H}_{1 k} \mathbf{F}_{k} \mathbf{F}_{k}^{H} \mathbf{H}_{1 k}^{H}
$$

denotes the interference covariance matrix of the macro UE. In the denominator of the right-hand side of (A.1), $\mathbf{G}_{1}^{H} \mathbf{Q}_{1} \mathbf{G}_{1}$ becomes a vanishing matrix because

$$
\begin{aligned}
\mathbf{G}_{1}^{H} \mathbf{Q}_{1} \mathbf{G}_{1} & =\mathbf{G}_{1}^{H}\left(\sum_{k \in \mathscr{B}_{1}} \frac{P_{1 k}}{\rho d} \mathbf{H}_{1 k} \mathbf{F}_{k} \mathbf{F}_{k}^{H} \mathbf{H}_{1 k}^{H}\right) \mathbf{G}_{1} \\
& =\sum_{k \in \mathscr{B}_{1}} \frac{P_{1 k}}{\rho d}\left(\mathbf{G}_{1}^{k} \mathbf{H}_{1 k} \mathbf{F}_{k} \mathbf{F}_{k}^{H} \mathbf{H}_{1 k}^{H} \mathbf{G}_{1}\right)=\mathbf{0}
\end{aligned}
$$

by (3). Then, $C_{M}(\rho)$ can be rewritten as

$$
\begin{aligned}
& C_{M}(\rho) \\
& \quad=\mathbb{E}\left[\log _{2} \operatorname{det}\left\{\mathbf{I}_{d}+\frac{\rho}{d} \mathbf{G}_{1}^{H} \mathbf{H}_{11} \mathbf{F}_{1} \mathbf{F}_{1}^{H} \mathbf{H}_{11}^{H} \mathbf{G}_{1}\right\}\right] .
\end{aligned}
$$

Substituting $\overline{\mathbf{H}}=\mathbf{G}_{1}^{H} \mathbf{H}_{11} \mathbf{F}_{1}$ into $C_{M}(\rho)$,

$$
\begin{aligned}
C_{M}(\rho) & =\mathbb{E}\left[\log _{2} \operatorname{det}\left\{\mathbf{I}_{d}+\frac{\rho}{d} \overline{\mathbf{H}} \overline{\mathbf{H}}^{H}\right\}\right] \\
& =E\left[\sum_{i=1}^{d} \log _{2}\left(1+\frac{\rho \lambda_{i}}{d}\right)\right],
\end{aligned}
$$


where $\lambda_{i}$ denotes the $i$ th largest eigenvalue of $\overline{\mathbf{H}}$. By the definition of the DoF,

$$
\begin{aligned}
\lim _{\rho \rightarrow \infty} \frac{C_{M}(\rho)}{\log _{2}(\rho)} & =\lim _{\rho \rightarrow \infty} \mathbb{E}\left[\sum_{i=1}^{d} \frac{\log _{2}\left(1+\rho \lambda_{i} / d\right)}{\log _{2}(\rho)}\right] \\
& =\lim _{\rho \rightarrow \infty} \mathbb{E}\left[\sum_{i=1}^{d} \frac{\log _{2}\left(\rho \lambda_{i} / d\right)+\mathcal{O}(1)}{\log _{2}(\rho)}\right] \\
& =\lim _{\rho \rightarrow \infty} \sum_{i=1}^{d} \frac{\mathbb{E}\left[\log _{2}\left(\rho \lambda_{i} / d\right)+\mathcal{O}(1)\right]}{\log _{2}(\rho)} \\
& \approx \lim _{\rho \rightarrow \infty} \sum_{i=1}^{d} \frac{\log _{2}(\rho)+\mathcal{O}(1)}{\log _{2}(\rho)} \\
& \approx \lim _{\rho \rightarrow \infty} \frac{d \log _{2}(\rho)+\mathcal{O}(1)}{\log _{2}(\rho)}=d .
\end{aligned}
$$

\section{B. Proof of Lemma 3}

Let us consider an arbitrary complex matrix $\mathbf{A} \in \mathbb{C}^{m \times n}$ and a unitary matrix $\mathbf{R} \in \mathbb{C}^{m \times p}$. If $\mathbf{R}$ is square, that is, $p=m, \mathbf{R}^{H} \mathbf{A}$ can be regarded as the rotation of $\mathbf{A}$. Then, the singular values of $\mathbf{R}^{H} \mathbf{A}$ are the same as $\mathbf{A}$. If $p<m$, however, $\operatorname{rank}\left(\mathbf{R}^{H} \mathbf{A}\right)<$ $\operatorname{rank}(\mathbf{A})$ and those singular values may be varied. In this case, to obtain the same singular values of $\mathbf{A}$, the columns of $\mathbf{R}^{H} \mathbf{A}$ should be the left singular vectors of $\mathbf{A}$.

For the case that $M<2 N$, the achievable DoF is $d=$ $\min (M / 2, N)<M$ and $N$, and $\mathbf{G}_{1}$ in (10) is a nonsquare $N \times$ $d$ matrix. Therefore, for optimal $\mathbf{F}_{1}$, the columns of $\mathbf{G}_{1}$ should be the left singular vectors of $\mathbf{H}_{11}$. For $M \geq 2 N$, the achievable DoF is $d=N$, and $\mathbf{G}_{1}$ is a square $N \times N$ matrix. In this case, $\mathbf{F}_{1}$ is always optimal for any unitary square matrix $\mathbf{G}_{1}$ because rank and singular values of $\mathbf{G}_{1}^{H} \mathbf{H}_{11}$ are invariant to those of $\mathbf{H}_{11}$. Therefore, the proposed MCP-IA is always optimal for the macro UE for any antenna configuration with $M \geq 2 N$.

\section{Competing Interests}

The authors declare that there is no conflict of interests regarding the publication of this paper.

\section{Acknowledgments}

This research was a part of the project titled "Development of Distributed Underwater Monitoring \& Control Networks," funded by the Ministry of Oceans and Fisheries, Republic of Korea.

\section{References}

[1] V. Chandrasekhar, J. G. Andrews, and A. Gatherer, "Femtocell networks: a survey," IEEE Communications Magazine, vol. 46, no. 9, pp. 59-67, 2008.

[2] C. Patel, M. Yavuz, and S. Nanda, "Femtocells [industry perspectives]," IEEE Wireless Communications, vol. 17, no. 5, pp. 6-7, 2010.
[3] A. Damnjanovic, J. Montojo, Y. Wei et al., "A survey on 3GPP heterogeneous networks," IEEE Wireless Communications, vol. 18, no. 3, pp. 10-21, 2011.

[4] B. Soret, H. Wang, K. I. Pedersen, and C. Rosa, "Multicell cooperation for LTE-advanced heterogeneous network scenarios," IEEE Wireless Communications, vol. 20, no. 1, pp. 27-34, 2013.

[5] D. López-Pérez, I. Güvenç, G. De La Roche, M. Kountouris, T. Q. S. Quek, and J. Zhang, "Enhanced intercell interference coordination challenges in heterogeneous networks," IEEE Wireless Communications, vol. 18, no. 3, pp. 22-30, 2011.

[6] I. Hwang, C.-B. Chae, J. Lee, and R. W. Heath Jr., "Multicell cooperative systems with multiple receive antennas," IEEE Wireless Communications, vol. 20, no. 1, pp. 50-58, 2013.

[7] S. Sun, Q. Gao, Y. Peng, Y. Wang, and L. Song, "Interference management through CoMP in 3GPP LTE-advanced networks," IEEE Wireless Communications, vol. 20, no. 1, pp. 59-66, 2013.

[8] V. R. Cadambe and S. A. Jafar, "Interference alignment and degrees of freedom of the $K$-user interference channel," IEEE Transactions on Information Theory, vol. 54, no. 8, pp. 34253441, 2008.

[9] C. M. Yetis, T. Gou, S. A. Jafar, and A. H. Kayran, "On feasibility of interference alignment in MIMO interference networks," IEEE Transactions on Signal Processing, vol. 58, no. 9, pp. 47714782, 2010.

[10] L. Ruan, V. K. N. Lau, and M. Z. Win, “The feasibility conditions for interference alignment in MIMO networks," IEEE Transactions on Signal Processing, vol. 61, no. 8, pp. 2066-2077, 2013.

[11] K. Gomadam, V. R. Cadambe, and S. A. Jafar, "A distributed numerical approach to interference alignment and applications to wireless interference networks," IEEE Transactions on Information Theory, vol. 57, no. 6, pp. 3309-3322, 2011.

[12] D. C. Moreira, Y. C. B. Silva, K. Ardah, W. C. Freitas, and F. R. P. Cavalcanti, "Convergence analysis of iterative interference alignment algorithms," in Proceedings of the International Telecommunications Symposium (ITS '14), pp. 1-5, São Paulo, Brazil, August 2014.

[13] S. Chen and R. S. Cheng, "Clustering for interference alignment in multiuser interference network," IEEE Transactions on Vehicular Technology, vol. 63, no. 6, pp. 2613-2624, 2014.

[14] B. Guler and A. Yener, "Selective interference alignment for MIMO cognitive femtocell networks," IEEE Journal on Selected Areas in Communications, vol. 32, no. 3, pp. 439-450, 2014.

[15] D. Castanheira, A. Silva, and A. Gameiro, "Set optimization for efficient interference alignment in heterogeneous networks," IEEE Transactions on Wireless Communications, vol. 13, no. 10, pp. 5648-5660, 2014.

[16] G. Gupta and A. K. Chaturvedi, "User selection in MIMO interfering broadcast channels," IEEE Transactions on Communications, vol. 62, no. 5, pp. 1568-1576, 2014.

[17] H. Okamoto, K. Kitao, and S. Ichitsubo, "Outdoor-to-indoor propagation loss prediction in $800-\mathrm{MHz}$ to $8-\mathrm{GHz}$ band for an urban area," IEEE Transactions on Vehicular Technology, vol. 58, no. 3, pp. 1059-1067, 2009.

[18] S. Y. Lim, Z. Yun, and M. F. Iskander, "Propagation measurement and modeling for indoor stairwells at 2.4 and $5.8 \mathrm{GHz}$," IEEE Transactions on Antennas and Propagation, vol. 62, no. 9, pp. 4754-4761, 2014.

[19] G. R. MacCartney, T. S. Rappaport, S. Sun, and S. Deng, "Indoor office wideband millimeter-wave propagation measurements and channel models at 28 and $73 \mathrm{GHz}$ for ultra-dense $5 \mathrm{G}$ wireless networks," IEEE Access, vol. 3, pp. 2388-2424, 2015. 
[20] V. Chandrasekhar, M. Kountouris, and J. G. Andrews, "Coverage in multi-antenna two-tier networks," IEEE Transactions on Wireless Communications, vol. 8, no. 10, pp. 5314-5327, 2009.

[21] D. C. Moreira, Y. C. B. Silva, K. Ardah, W. C. Freitas Jr., and F. R. P. Cavalcanti, "Convergence analysis of iterative interference alignment algorithms," in Proceedings of the International Telecommunications Symposium (ITS '14), São Paulo, Brazil, August 2014

[22] D. Samardzija and N. Mandayam, "Pilot-assisted estimation of MIMO fading channel response and achievable data rates," IEEE Transactions on Signal Processing, vol. 51, no. 11, pp. 28822890, 2003.

[23] S. J. Lee, "Effect of least square channel estimation errors on achievable rate in MIMO fading channels," IEEE Communications Letters, vol. 11, no. 11, pp. 862-864, 2007.

[24] 3GPP TR 36.814 ver. 9.0.0, "Evolved Universal Terrestrial Radio Access (E-UTRA); Further advancements for E-UTRA physical layer aspects," March 2010.

[25] R. S. Blum, "MIMO capacity with interference," IEEE Journal on Selected Areas in Communications, vol. 21, no. 5, pp. 793-801, 2003.

[26] G. H. Golub and C. F. Van Loan, Matrix Computations, Johns Hopkins Studies in the Mathematical Sciences, The Johns Hopkins University Press, Baltimore, Md, USA, 3rd edition, 1996.

[27] K. Zu and R. C. de Lamare, "Low-complexity lattice reductionaided regularized block diagonalization for MU-MIMO systems," IEEE Communications Letters, vol. 16, no. 6, pp. 925-928, 2012.

[28] K. Zu, R. C. de Lamare, and M. Haardt, "Generalized design of low-complexity block diagonalization type precoding algorithms for multiuser MIMO systems," IEEE Transactions on Communications, vol. 61, no. 10, pp. 4232-4242, 2013. 

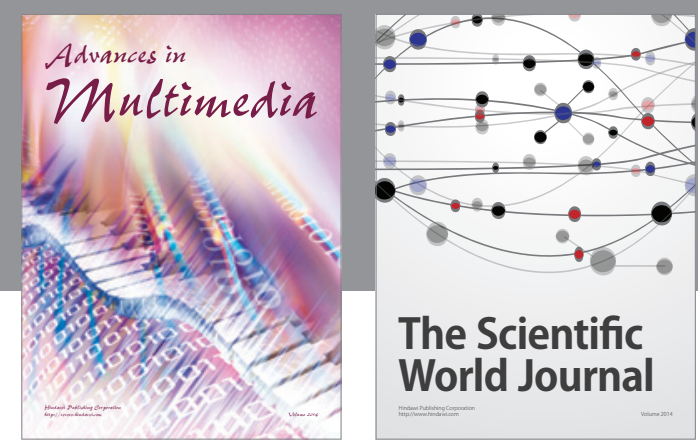

The Scientific World Journal
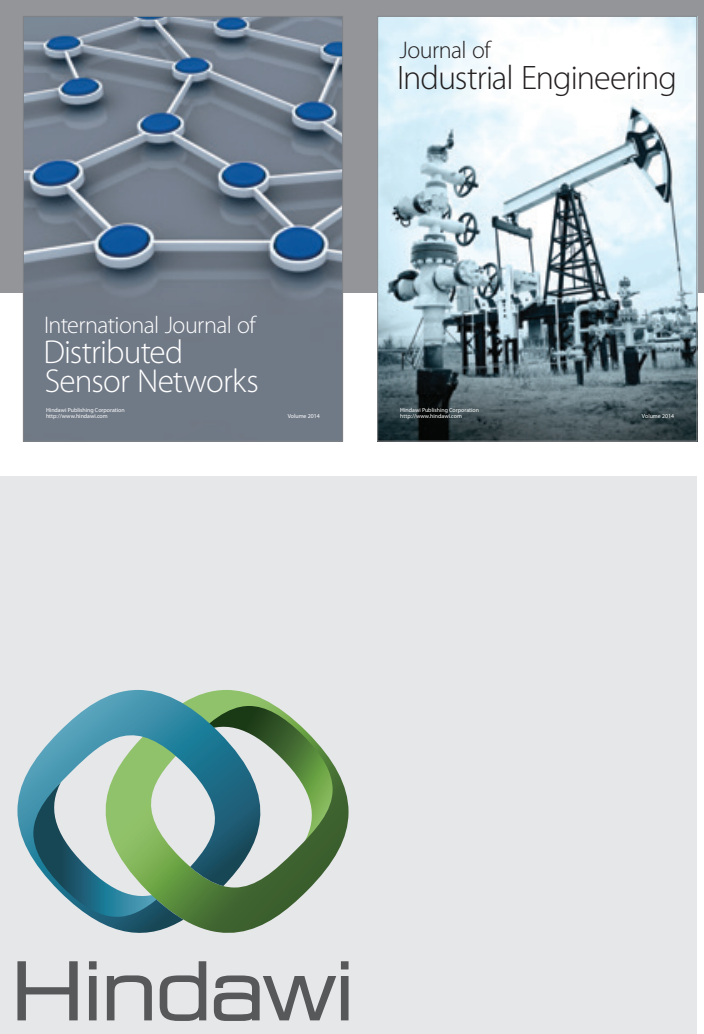

Submit your manuscripts at

https://www.hindawi.com

\section{Computer Networks} and Communications
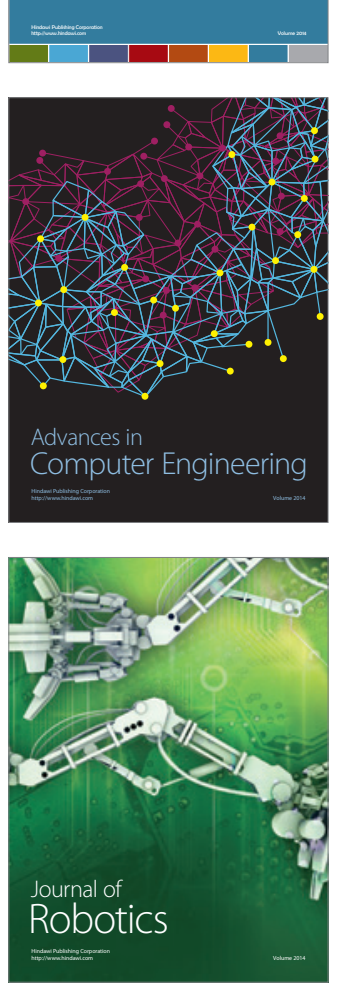
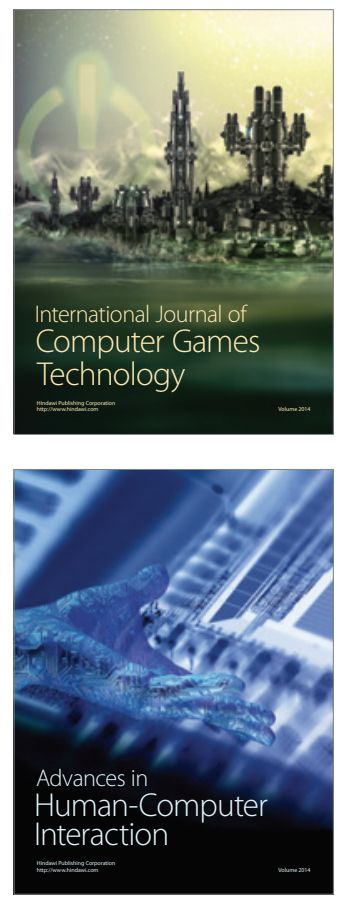
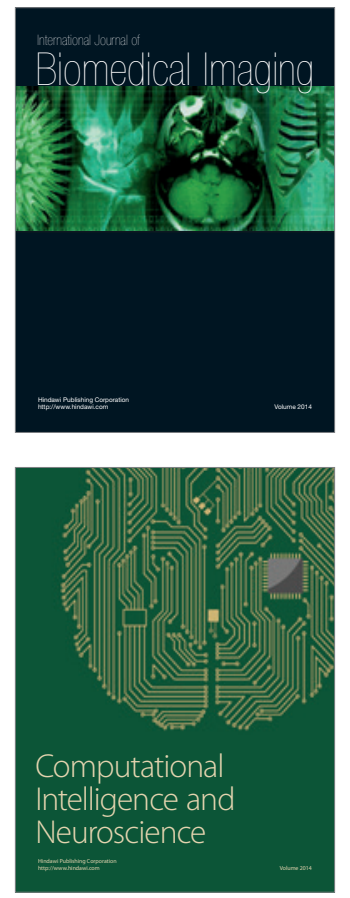
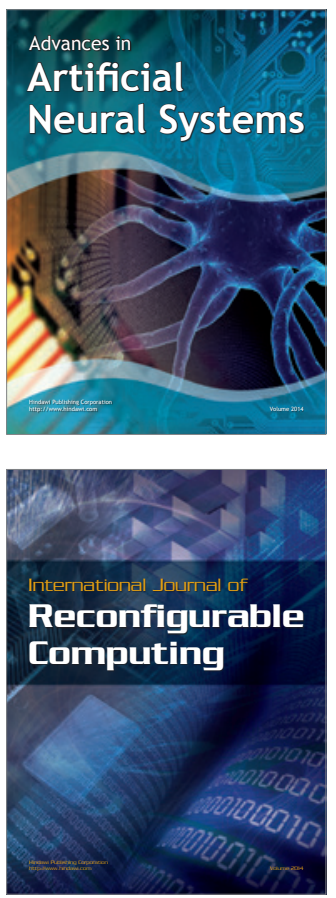
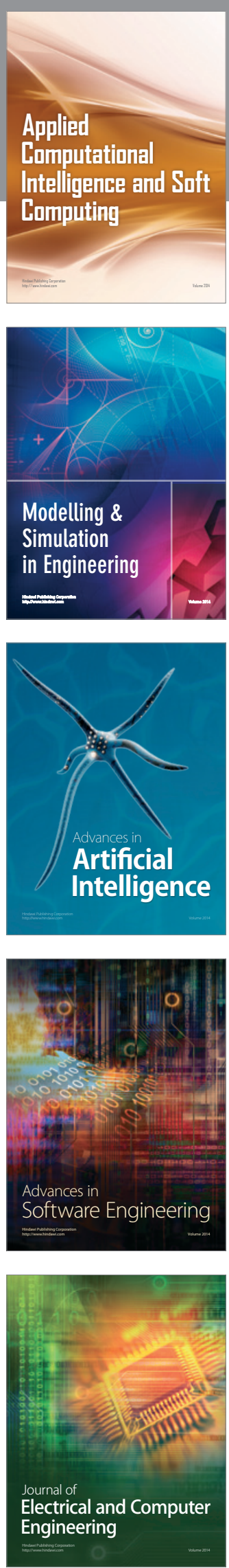